\title{
Effectiveness of Pen Pressure, Azimuth, and Altitude Features for Online Signature Verification
}

\author{
Daigo Muramatsu $^{1}$ and Takashi Matsumoto ${ }^{2}$ \\ ${ }^{1}$ Department of Electrical and Mechanical Engineering, Seikei University, \\ 3-3-1 Kichijoji-kitamachi, Musashino-shi, Tokyo 180-8633, Japan \\ muramatsu@st.seikei.ac.jp \\ 2 Department of Electrical Engineering and Bioscience, Waseda University, \\ 3-4-1 Okubo, Shinjuku-ku, Tokyo 169-8555, Japan \\ takashi@mse.waseda.ac.jp
}

\begin{abstract}
Many algorithms for online signature verification using multiple features have been proposed. Recently it has been argued that pen pressure, azimuth, and altitude can cause instability and deteriorate the performance. Algorithms without pen pressure and inclination features outperformed with them in SVC2004. However, we previously found that these features improved the performance in evaluations using our private database. The effectiveness of the features thus depended on the algorithm. Therefore, we re-evaluated our algorithm using the same database as used in SVC2004 and discuss the effectiveness of pen pressure, azimuth and altitude. Experimental results show that even though these features are not so effective when they are used by themselves, they improved the performance when used in combination with other features. When pen pressure and inclination features were considered, an EER of 3.61\% was achieved, compared to an EER of $5.79 \%$ when these features were not used.
\end{abstract}

Keywords: Online signature verification, Pen pressure, Pen azimuth, Pen altitude, Fusion, SVC2004.

\section{Introduction}

Recently, the renewed interest in biometric authentication has resulted in its application to many situations. Several biometric authentication methods have been proposed and studied; however, no perfect method currently exists. The suitability of the method depends on the situation, as well as the required security level. Online signature verification is a promising candidate as an authentication method for several reasons. First, handwritten signatures are widely accepted as the authentication method in many countries for various purposes, such as authorizing credit card and banking transactions and signing agreements and legal documents. 
Second, because online signature verification can incorporate dynamic information about the handwritten signature, it can often achieve higher performance than static signatures 1. Moreover, since it is difficult to extract dynamic information from a static signature, it is more difficult to forge. Finally, a person can modify his or her signature if it is stolen. This is a notable feature because physiological biometrics such as fingerprints or irises cannot be modified or renewed. Therefore, online signature verification is a promising candidate as an authentication method. However, online signature verification is not perfect, and it is important to develop algorithms that can achieve high performance.

Many algorithms have been proposed for online signature verification, using multiple features acquired using various types of devices. Some special devices $[2]-$ [4] and techniques [5] for doing so have been proposed; however, popular devices for online signature verification are tablets (graphic tablets), Tablet PCs, and PDAs. The features that can be obtained depend on the type of device; for example, only pen position and pen up/down information are available using PDAs, whereas pen pressure features are also available when using Tablet PCs. Moreover, some tablets can acquire pen inclination information, like pen altitude and azimuth. Some public databases for online signature verification, for example, BIOMET 6], MCYT [], and SVC [8, were prepared using data captured from tablets. Thus, information about pen position, pen pressure, pen altitude, and pen azimuth is available 1 Researchers who use these databases generally use all of the information in the database 9-14. However, it has argued that using the pressure, azimuth, and altitude can cause instability and deteriorate the performance [8] [15]- 17].

The First International Signature Verification Competition (SVC) was held in 2004; there were two tasks in the competition, Task 1 and Task 2. Participants could use pen position, pressure, azimuth, and altitude in Task 2, though only pen position and pen up/down information were available in Task 1 . The results of Task 1 outperformed those of Task 2 [8. As a result, some researchers have stopped using pen pressure, 15, pen altitude, and pen azimuth 16] or have expressed concern about using pen inclination features [17. On the other hand, some researchers have proposed algorithms taking account of pen inclination features and reported improved performance $18-20$. We also reported improved performance using pen pressure and inclination features [14. However, the databases used by researchers for evaluation were not same. Thus, we evaluated an algorithm using the same database as that used in SVC2004 and report the results in this paper.

We performed several experiments, and the results show that our algorithm could improve the performance from an equal error rate (EER) of $5.79 \%$ to $3.61 \%$ for user-dependent threshold parameters, and from $12.67 \%$ to $10.15 \%$ for a global threshold parameter by using pen pressure, altitude, and azimuth features. Thus, the effectiveness of using these features depends on the type of algorithm.

${ }^{1}$ Information about pen pressure, pen altitude, and pen azimuth were not made available in Task 1 of the SVC database. 


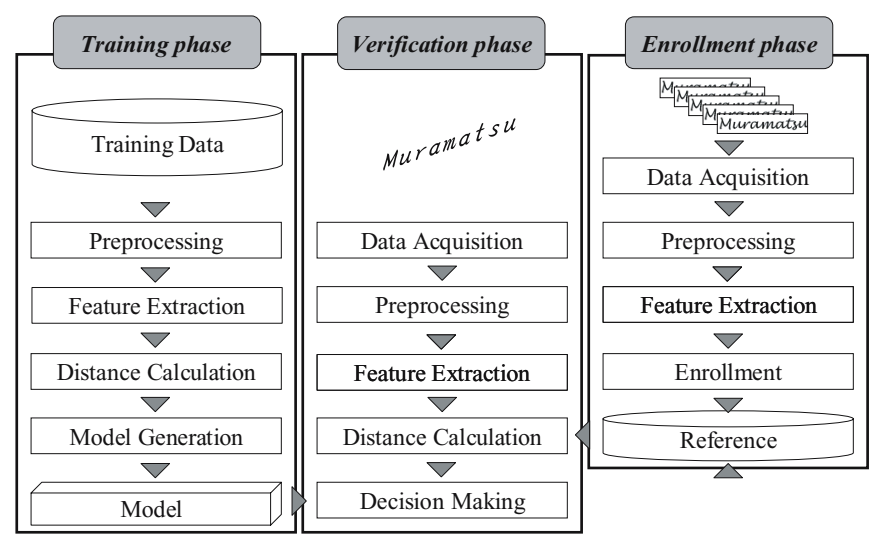

Fig. 1. Overall algorithm

\section{The Algorithm}

Figure 1 depicts our algorithm for online signature verification. There are three phases in the algorithm:

(i) Training phase

Signatures for training are provided in the training phase. After preprocessing and feature extraction, multiple distances between the training signatures are calculated. Parameters of a fusion model are estimated by using the distances from the training signatures.

(ii) Enrollment phase

In the enrollment phase, candidates of reference signatures are provided, together with the identity of the users (signers). After preprocessing and feature extraction, reference signatures are selected.

(iii) Verification phase

In the verification phase, a signature under question, with a claimed identity, is provided. After preprocessing and feature extraction, multiple distances between the signature under question and the reference signatures are calculated. Then, these multiple distances are combined in the fusion model and a decision is made.

The training, enrollment, and verification phases involve all or some of the following stages: (a) data acquisition, (b) pre-processing, (c) feature extraction, (d) enrollment, (e) similarity/distance calculation, (f) model generation, and (g) decision making. The boundaries between the stages are not rigid; therefore, one stage can be included in another stage. Only the feature extraction, distance calculation, and decision making stages are explained in this paper. Details of our signature verification algorithm were given in reference 11] and reference 14 . 


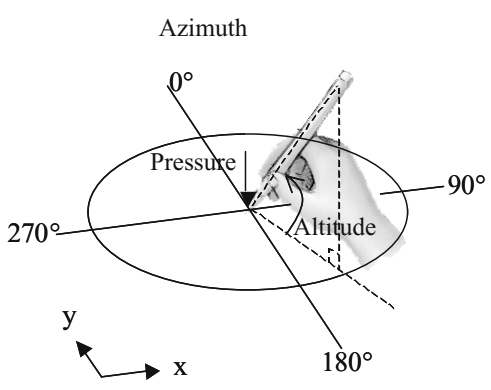

Fig. 2. Data from the tablet
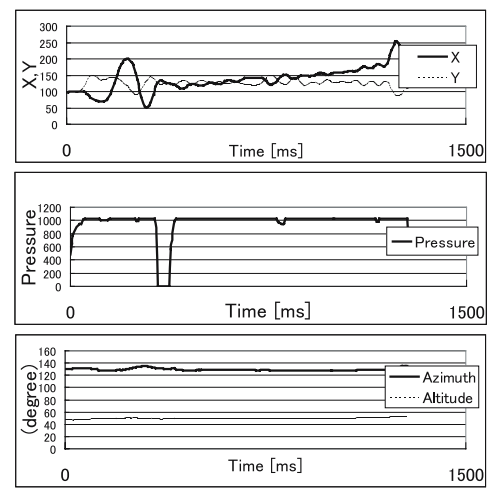

Fig. 3. Top: Trajectories of $\mathrm{x}$ and $\mathrm{y}$ pen positions. Middle: Trajectory of pen pressure. Bottom: Trajectories of azimuth and altitude.

\subsection{Feature Extraction}

Raw data from the tablet consists of five-dimensional time-series data:

$$
\begin{array}{r}
\text { Signature }=(x(j), y(j), p(j), \psi(j), \phi(j)) \\
j=1,2, \ldots \ldots, J
\end{array}
$$

Here, $(x(j), y(j))$ is the pen position; $p(j)$, the pen pressure; $\psi(j)$, the azimuth; and $\phi(j)$, the altitude of the pen at time $j$ (depicted in Figs. 2 and 3 ). The following features are extracted from the raw data.

$$
\begin{aligned}
X(j) & =\frac{x(j)-x_{g}}{x_{\max }-x_{\min }} \\
Y(j) & =\frac{y(j)-y_{g}}{y_{\max }-y_{\min }} \\
|V|(j) & =\sqrt{V_{x}^{2}(j)+V_{y}^{2}(j)} \\
\theta_{V}(j) & =\tan ^{-1} \frac{V_{y}(j)}{V_{x}(j)} \\
\text { where } & V_{x}(j)=X(j+1)-X(j), V_{y}(j)=Y(j+1)-Y(j)
\end{aligned}
$$

where $\left(x_{g}, y_{g}\right)$ is the centroid of the signature, and $x_{\min }, y_{\min }$ and $x_{\max }, y_{\max }$ are the minimum and maximum values of $x(j), y(j)$. In addition to the above features, pen pressure $p(j)$, azimuth $\psi(j)$, and altitude $\phi(j)$ are used as features. Although other features proposed in reference 16] 21-23] can be useful, our goal is to discuss the effectiveness of pen pressure, azimuth, and altitude. Thus, we use only seven features in this paper. 


\subsection{Distance Calculation}

Our approach calculates multiple distances from multiple features using dynamic programming. The distance of each feature was calculated independently. Thus, the distance vector between two signatures, Rsig and Sig, is

$$
\operatorname{Dist}(R \operatorname{sig}, \text { Sig })=\left(D_{X}, D_{Y}, D_{|V|}, D_{\theta_{V}}, D_{p}, D_{\psi}, D_{\phi}\right) .
$$

\subsection{Decision Making}

Our fusion model calculates the following score:

$$
\operatorname{Score}(\operatorname{Sig} ; I D)=\sum_{m=1}^{M} f\left(X\left(\operatorname{Rsig}_{I D_{m}}, \operatorname{Sig}\right), \operatorname{Mean}_{I D} ; \Theta\right),
$$

where $M$ is a number of reference signatures and $X$ is the input vector to the fusion model, described by

$$
\begin{aligned}
& X\left(\operatorname{Rsig}_{I D_{m}}, S i g\right) \\
& =\left(\frac{D_{1}\left(R \operatorname{Rig}_{I D_{m}}, S i g\right)}{Z_{1} T_{m}}, . ., \frac{D_{i}\left(R \operatorname{Rsi}_{I D_{m}}, S i g\right)}{Z_{i} T_{m}}, . ., \frac{D_{N}\left(R \operatorname{Rig}_{I D_{m}}, S i g\right)}{Z_{N} T_{m}} .\right.
\end{aligned}
$$

Here, $D_{i}$ is a distance calculated with the $i_{t h}$ feature, $T_{m}$ is the duration of the reference signature, $Z_{i}$ is a normalization constant calculated from a training dataset, and $N$ is a dimension of input vector $X$. $\operatorname{Mean}_{I D}$ is a mean vector defined by

$$
\begin{gathered}
\operatorname{Mean}_{I D}=\left(\operatorname{mean}_{1}^{I D}, . ., \operatorname{mean}_{i}^{I D}, . ., \operatorname{mean}_{N}^{I D}\right) \\
\operatorname{mean}_{i}^{I D}=\frac{1}{M^{2}} \sum_{m=1}^{M} \sum_{n=1}^{M} \frac{D_{i}\left(\operatorname{Rsig}_{I D_{m}}, \operatorname{Rsig}_{I D_{n}}\right)}{Z_{i} T_{m}},
\end{gathered}
$$

and $\Theta$ is a parameter set for the fusion model estimated from the training dataset.

A final decision is made by the following rule:

$$
\text { Signature is }\left\{\begin{array}{l}
\text { Accept if } \operatorname{Score}(\text { Sig; ID }) \geq \text { Threshold }(c) \\
\text { Reject if } \operatorname{Score}(\text { Sig; ID })<\text { Threshold }(c)
\end{array}\right.
$$

where $c$ is a parameter for adjusting the threshold value.

\section{Experiments}

Two experiments are described in this paper:

(i) Experiment 1: Performance evaluation of each distance from a feature; and

(ii) Experiment 2: Performance evaluation of fusion models that combine different distances.

The following three databases were used for evaluating the performance: 


\section{BIOMET}

This database was collected by Garcia-Salicetti et al. [6]. From this database, signatures for 61 persons were used in our experiments. For each person, five genuine signatures were used as reference signatures, and ten genuine signatures from a second session and twelve skillfully forged signatures were used for evaluation. This database was used for training the parameters of the fusion model in Experiment 2 and was used for evaluation only in Experiment1.

\section{MCYT}

This database was collected by Ortega-Garcia et al. [7. Signatures from 100 persons were used for evaluation in our experiments. For each person, five genuine signatures were used as reference signatures, and twenty genuine signatures and twenty-five skillfully forged signatures were used for evaluation.

\section{SVC}

This database was collected by Yeung et al. 8 and was used for the First International Signature Verification Competition. This database is formed of two databases for task 1 and task 2 . In task 2, pen position, pressure, and inclination features are available, whereas in task 1, only pen position and pen up/down features are available. Five genuine signatures randomly selected from a first session were used as reference signatures, and ten genuine signatures from a second session and twenty skillfully forged signatures were used for evaluation in one experiment. We considered ten combinations of five genuine signatures as reference signatures, and performed ten experiments while changing only the reference signatures. These were the same conditions as in the competition and the study described in reference 24.

\subsection{Experiment 1}

The three public databases described above, BIOMET, MCYT, and SVC (task 1 and task 2), were used to evaluate the performance. To evaluate the effectiveness of each feature, a one-dimensional distance score was calculated:

$$
D^{\prime}(S i g ; I D, i)=\frac{1}{M} \sum_{m=1}^{M} \frac{D_{i}\left(R_{s i g_{I} D_{m}}, S i g\right)}{T_{m}} .
$$

Table 1. EER for each distance measure (global threshold parameter)

\begin{tabular}{c|c|cccc}
\hline No. & Feature & \multicolumn{4}{|c}{ MCYT } \\
\hline 1 & $X$ & 10.5 & 9.39 & 24.1 & 22.6 \\
2 & $Y$ & 7.7 & 9.54 & 17.8 & 20.2 \\
3 & $|V|$ & 7.2 & 7.32 & 16.9 & 16.9 \\
4 & $\theta_{V}$ & 7.8 & 6.57 & 16.1 & 17.6 \\
7 & $P$ & 16.1 & 23.24 & - & 24.6 \\
8 & $\psi$ & 23.3 & 21.41 & - & 31.6 \\
9 & $\phi$ & 27.0 & 24.58 & - & 32.2 \\
\hline
\end{tabular}


Then, a decision was made based on

$$
\text { Signature }=\left\{\begin{array}{l}
\text { Accept if } D^{\prime}(\text { Sig; ID, } i)<\text { Threshold }_{D_{i}}(c) \\
\text { Reject if } D^{\prime}(\text { Sig; ID.i }) \geq \text { Threshold }_{D_{i}}(c)
\end{array} .\right.
$$

The experimental results are summarized in Table 1 . The pen altitude and azimuth did not produce good results; pressure was better than both altitude and azimuth but was worse than pen position and velocity features.

\section{$3.2 \quad$ Experiment2}

The BIOMET database was used for training the fusion model parameters, and the MCYT and SVC (task 1 and task 2) databases were used for evaluation.

Table 2. EER of fusion model

\begin{tabular}{c|ccc|ccc}
\hline Threshold & \multicolumn{3}{|c|}{ User-dependent } & \multicolumn{3}{c}{ Global } \\
\hline Feature set & MCYT & $\begin{array}{c}\text { SVC } \\
\text { (task 1) }\end{array}$ & $\begin{array}{c}\text { SVC } \\
\text { task 2) }\end{array}$ & MCYT & $\begin{array}{c}\text { SVC } \\
\text { (task 1) }\end{array}$ & $\begin{array}{c}\text { SVC } \\
\text { task 2) }\end{array}$ \\
\hline Setting 1 & 3.05 & 7.46 & 10.7 & 6.30 & 15.04 & 15.38 \\
Setting 2 & 2.23 & 4.74 & 5.79 & 4.91 & 11.67 & 12.67 \\
Setting 3 & 1.75 & - & 5.45 & 3.93 & - & 10.35 \\
Setting 4 & 1.89 & - & 4.57 & 4.32 & - & 11.58 \\
Setting 5 & 2.29 & - & 4.32 & 4.50 & - & 11.52 \\
Setting 6 & 1.50 & - & 4.56 & 3.52 & - & 10.38 \\
Setting 7 & 1.75 & - & 4.30 & 3.58 & - & 10.38 \\
Setting 8 & 2.00 & - & 3.53 & 4.44 & - & 10.69 \\
Setting 9 & 1.57 & - & 3.61 & 3.72 & - & 10.15 \\
\hline
\end{tabular}

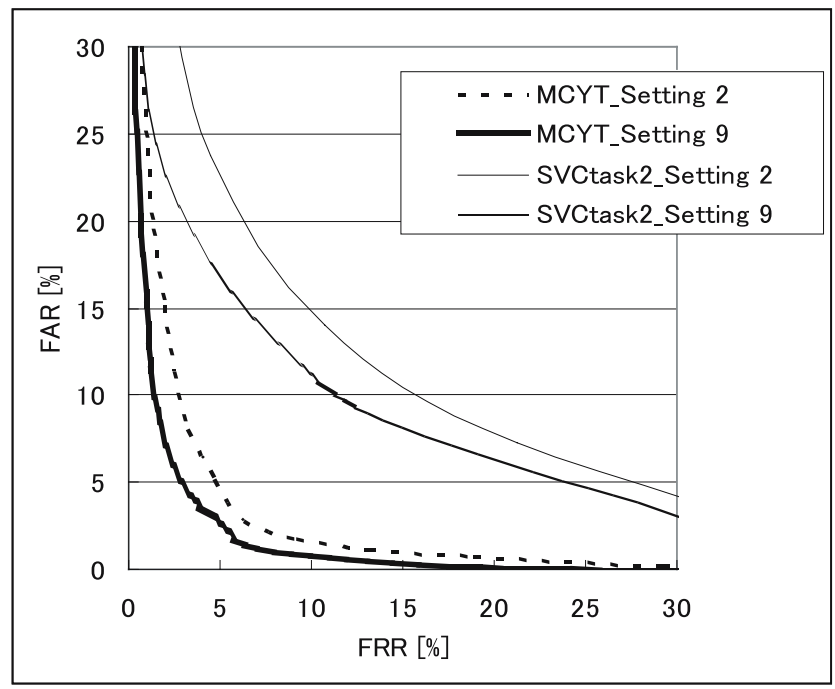

Fig. 4. Error trade-off curves of fusion model for MCYT and SVC (task 2) databases 
The following combinations of distances were evaluated:

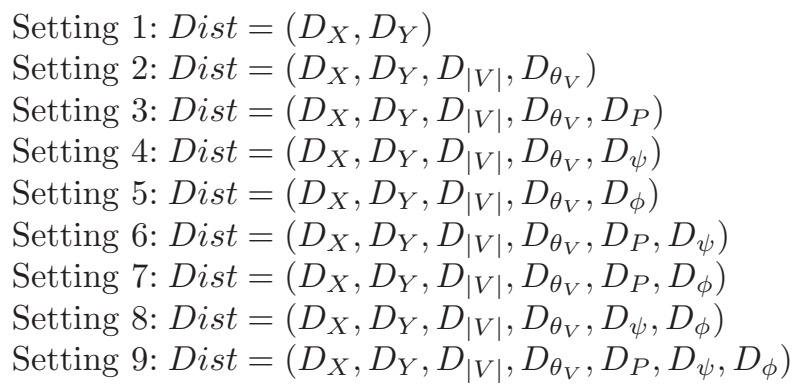

Equal error rates were obtained with both user-dependent threshold parameters and a global threshold parameter (results summarized in Table 2). EER was calculated using the methods of Fingerprint Verification Competition (FVC) 25]. Figure4 shows the error trade-off curve of Setting 2 and Setting 9 for the MCYT and SVC (task 2) databases with a global threshold parameter. An error trade-off curve shows the relation between false rejection rate (FRR) and false acceptance rate (FAR). Judging from the results of Settings 2, 3, 4, and 5, pen pressure, azimuth, and altitude features contributed to improved performance.

\section{Conclusion}

We evaluated our online signature verification algorithm using the BIOMET, MCYT, and SVC public databases. It has recently been argued that pen pressure, azimuth, and altitude can cause instability and deteriorate the performance of online signature verification, because an algorithm that used only pen position features won a previous competition (SVC2004). We evaluated our algorithm using the same databases that were used for that competition. Our experimental results show that, by incorporating both pen pressure and inclination features, we could improve the performance from EER of $5.79 \%$ to $3.61 \%$ for SVC task 2 with user-dependent threshold parameters, and from EER of $12.67 \%$ to $10.15 \%$ with a global threshold parameter. Even though these features are not so effective when they are used by themselves, they improved the performance when used together with other features. Moreover, our results outperformed the best result published on the SVC websit 2 and reported in [24].

We only evaluated a few features in this study; combinations of many more features, such as those proposed in references[16] [21]-23], should improve the performance. Also, more efficient score normalization algorithm will be necessary because the difference between user-dependent threshold parameters and a global threshold parameter was large. This will be the subject of our future work.

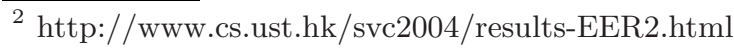




\section{Acknowledgements}

The authors are grateful to J. Ortega-Garcia and J. Fierrez-Aguilar of Universidad Politecnica de Madrid, Madrid, Spain, and to B. Dorizzi and S. GarciaSalicetti of Institut National Des Telecommunications, Evry, France for providing us with the databases. The authors would also like to thank D.Y. Yeung for advice on the evaluation method used in SVC2004. This work was supported by Grants-in-Aid for Scientific Research from Ministry of Education, Culture, Sports, Science and Technology.

\section{References}

1. Plamondon, R., Lorette, G.: Automatic signature verification and writer identification - The state of the art. Pattern Recognition 22(2), 101-131 (1989)

2. Martens, R., Claesen, L.: Incorporating local consistency information into the online signature verification process. IJDAR 1(2), 110-115 (1998)

3. Shimizu, H., Kiyono, S., Motoki, T., Gao, W.: An electrical pen for signature verification using a two-dimensional optical angle sensor. Sensor and Actuators A111, 211-216 (2004)

4. Hook, C., Kempf, J., Scharfenberg, G.: A Novel digitizing pen for the analysis of pen pressure and inclination in handwriting biometrics. In: Maltoni, D., Jain, A.K. (eds.) BioAW 2004. LNCS, vol. 3087, pp. 283-294. Springer, Heidelberg (2004)

5. Munich, M.E., Perona, P.: Visual identification by signature tracking. IEEE Trans. Pattern Anal. and Machine Intell. 25(2), 200-217 (2003)

6. Garcia-Salicetti, S., Beumier, C., Chollet, G., Dorizzi, B., Leroux-Les Jardins, J., Lunter, J., Ni, Y., Petrovska-Delacretaz, D.: BIOMET: a multimodal person authentication database including face, voice, fingerprint, hand and signature modalities. In: Kittler, J., Nixon, M.S. (eds.) AVBPA 2003. LNCS, vol. 2688, pp. 845-853. Springer, Heidelberg (2003)

7. Ortega-Garcia, J., Fierrez-Aguilar, J., Simon, D., Gonzalez, J., Faundez-Zanuy, M., Espinosa, V., Satue, A., Hernaez, I., Igarza, J.-J., Vivaracho, C., Escudero, D., Moro, Q.-I.: MCYT baseline corpus: a bimodal biometric database. IEE Proceedings Vision, Image and Signal Processing 150(6), 395-401 (2003)

8. Yeung, D.-Y., Chang, H., Xiong, Y., George, S., Kashi, R., Matsumoto, T., Rigoll, G.: SVC. First international signature verification competition. In: Zhang, D., Jain, A.K. (eds.) ICBA 2004. LNCS, vol. 3072, pp. 16-22. Springer, Heidelberg (2004)

9. Ortega-Garcia, J., Fierrez-Aguilar, J., Martin-Rello, J., Gonzalez-Rodriguez, J.: Complete signal modeling and score normalization for function-based dynamic signature verification. In: Kittler, J., Nixon, M.S. (eds.) AVBPA 2003. LNCS, vol. 2688, pp. 658-667. Springer, Heidelberg (2003)

10. Van Ly, B., Garcia-Salicetti, S., Dorizzi, B.: Fusion of HMM's likelihood and viterbi path for on-line signature verification. In: Maltoni, D., Jain, A.K. (eds.) BioAW 2004. LNCS, vol. 3087, pp. 318-331. Springer, Heidelberg (2004)

11. Hongo, Y., Muramatsu, D., Matsumoto, T.: Modification on intersession variability in on-line signature verifier. In: Kanade, T., Jain, A., Ratha, N.K. (eds.) AVBPA 2005. LNCS, vol. 3546, pp. 455-463. Springer, Heidelberg (2005)

12. Muramatsu, D., Kondo, M., Sasaki, M., Tachibana, S., Matsumoto, T.: A Markov chain Monte Carlo algorithm for bayesian dynamic signature verification. IEEE Trans. Information Forensics and Security 1(1), 22-34 (2006) 
13. Marcos Faundez-Zanuy, M.: On-line signature recognition based on VQ-DTW. Pattern Recognition 40, 981-992 (2007)

14. Muramatsu, D., Matsumoto, T.: Online signature verification using user generic fusion model. IEICE Trans. J90-D(2), 450-459 (2007)

15. Kholmatov, A., Yanikoglu, B.: Identity authentication using improved online signature verification method. Pattern Recognition Letters 26(15), 2400-2408 (2005)

16. Fierrez-Aguilar, J., Nannil, L., Lopez-Peñalba, J., Ortega-Garcia, J., Maltoni, D.: An on-line signature verification system based on fusion of local and global information. In: Kanade, T., Jain, A., Ratha, N.K. (eds.) AVBPA 2005. LNCS, vol. 3546, pp. 523-532. Springer, Heidelberg (2005)

17. Lei, H., Govindaraju, V.: A comparative study on the consistency of features in online signature verification. Pattern Recognition Letters 26(15), 2483-2489 (2005)

18. Taguchi, H., Kiriyama, K., Tanaka, E., Fujii, K.: On-line recognition of handwritten signature by feature extraction of the pen movements. IEICE Trans. J71-D(5), 830840 (1988) (Japanese)

19. Komiya, Y., Ohishi, T., Matsumoto, T.: A pen input on-line signature verifier integration position, pressure and inclination trajectories. IEICE Trans. INF. \& SYST. E84-D(7), 833-838 (2001)

20. Hangai, S., Yamanaka, S., Hanamoto, T.: On-line signature verification based on altitude and direction of pen movement. In: Proc. ICME 2000, vol. 1, pp. 489-492 (2000)

21. Lee, L.L., Berger, T., Aviczer, E.: Reliable on-line human signature verification systems. IEEE Trans. Pattern Anal. and Machine Intell. 18(6), 643-647 (1996)

22. Nalwa, V.S.: Automatic on-line signature verification. Proc. IEEE 85(2), 215-239 (1997)

23. Jain, A.K., Griess, F.D., Connell, S.D.: On-line signature verification. Pattern Recognition 35(12), 2963-2972 (2002)

24. Fierrez-Aguilar, J., Krawczyk, S., Ortega-Garcia, J., Jain, A.K.: Fusion of local and regional approaches for on-line signature verification. In: Li, S.Z., Sun, Z., Tan, T., Pankanti, S., Chollet, G., Zhang, D. (eds.) IWBRS 2005. LNCS, vol. 3781, pp. 188-196. Springer, Heidelberg (2005)

25. Maio, D., Maltoni, D., Cappelli, R., Wayman, J.L., Jain, A.K.: FVC2000: Fingerprint verification competition. IEEE Trans. on Pattern Anal. Machine Intell. 24(3), 402-412 (2003) 